\title{
Context and goodness in a focusing task
}

\author{
M. C. KING, W. B. CRIST, and G. R. LOCKHEAD \\ Duke University, Durham, North Carolina 27706
}

In a focusing task, people respond positively to one stimulus and negatively to all other stimuli that occur. The task has been called focusing in recognition of the possibility that only the target stimulus is relevant to performance, and that what people do is to focus on some aspect of the target, such as its configuration. The present study used eight patterns, and people focused on each pattern in eight different experimental conditions. The stimulus set was selected to have good patterns (according to symmetry, subjective goodness, and free classification measures) that were similar to each other (according to similarity judgments) and poor patterns that were dissimilar from one another and from the good patterns. The results were that the good patterns were difficult (speed and accuracy) to classify, and the poor patterns were easy. The similarity between the target pattern and the other patterns in the total set, i.e., the context of each focused stimulus, predicted performance. These results support the idea that similarity judgments measure relevant aspects of context and that the effects of context on performance should not be overlooked.

Similarity relations among items of the stimulus set, as assessed by direct scaling techniques, reliably predict performance in several classification tasks using both patterns and words as stimuli (King, Gruenewald, \& Lockhead, 1978). This paper reports an investigation of whether similarity relations predict performance in a classification task, called focusing, for which this question has not previously been evaluated. The focusing task has recently been used (Garner, 1978) in attempts to understand better why certain stimuli in a set are easier to classify than are others (Garner \& Sutliff, 1974; Pomerantz, 1977; but see Lockhead \& King, 1977). The task requires observers to classify a particular stimulus (the target) into one category.

The name, "focusing," was assigned to the task to reflect the hypothesis that observers search for, or selectively attend to, the target stimulus or some aspect of it. Consider when the stimuli are patterns. According to the hypothesis, the response to a presented pattern results from a comparison between that stimulus and some template-like representation, which is held in memory, of the sought target. If a match occurs, a "yes" response is given; if a nonmatch occurs, a "no" response is made without further processing of the nontarget stimulus.

"Thus at present the most likely explanation of the ability of subjects to use a focusing strategy is that the focused stimulus is easy to hold in memory, that it can be used to provide an accurate anticipation of one stimulus, and that a yes-no decision can then be made without actual processing of the alternative stimuli, those in effect in the negative set. And the

Thank you, Vivian Alvarez, for collecting the data for Studies 2 and 3 . pattern of results with the discrimination tasks indicates that subjects do use a focusing strategy even in the simple two-stimuli discrimination tasks, at least as long as there is a stimulus which is easy to hold in memory" (Garner, 1978, p. 306). Garner (1978) and Pomerantz (1977) have concluded that focusing is easier for observers if the target pattern has a good rather than a poor configuration and that this is so because good patterns are easier to encode or to remember than are poor patterns. Accordingly the performance benefit of a focusing strategy for a particular pattern may depend primarily on physical properties which determine the configuration of that pattern.

This means that properties of the target stimulus in a focusing task should predict performance, and relations between the target and alternative stimuli in the set should be much less important, if important at all. Hence, while recognizing that the relations between stimuli are usually important in classification tasks, Garner discussed the importance of similarity relations to performance in the focusing task when the target has a good configuration and concluded that "similarity of the alternative stimuli to the focused stimulus is not very important because the subject presumably uses a go-no-go strategy" (1978, p. 306).

This assumption of a go/no-go response strategy has the advantage of being sufficiently specific to be testable. The subject initially focuses on or selectively attends to a particular feature, attribute, configuration, or object. When the stimulus occurs, the subject responds positively to the presence of the sought focus (go) or negatively to its absence (no-go). Positive responses should thereby be faster than negative responses, since the "go" response is evaluated first. 
Moreover, negative responses to all nontarget stimuli should be of approximately the same latency, since "no-go" is a default response. Further, the benefits associated with this strategy should be linked with the pattern goodness of the focused stimulus. Patterns of good configuration should be easy to focus, and patterns of poor configuration should be difficult.

An alternative hypothesis predicts classification performance to be a function of the similarity relations among task stimuli but does not address specific physical characteristics of individual stimulus members in a set (Lockhead, 1972). In a focusing task, the relations between a focused stimulus and the rest of the set should predict both positive and negative response times according to that hypothesis. Stimuli should be easy or difficult to focus on depending on whether they are dissimilar or similar to alternative stimuli. By this view, the goodness of the target is not sufficient to predict performance; the interrelations between task stimuli must be considered. If this is so, then the similarity hypothesis might account for the processing advantage which has been associated with good stimuli in classification tasks. This would occur if the good patterns that have previously been found easy to classify tended also to be dissimilar to other patterns in the stimulus sets investigated.

In a study relevant to these issues, Garner and Sutliff (1974) had observers discriminate between all possible pairs of six different patterns; there were 15 different conditions. The time to report which of two patterns had been presented was relatively short whenever the discrimination involved one good pattern and one poor pattern; response times were longer when both patterns were good; the times were longest when the two patterns were poor. Garner and Sutliff observed that this reflects the similarity difference between items; that performance was faster when the two patterns were dissimilar. They also reported that goodness was an important factor in their study. Within each condition (pair), the better stimulus was classified faster. We more formally similarity scaled their stimuli and found that, indeed, the correlation between the average of the dissimilarity ratings for each pair and the average time to classify members of the pair as reported by Garner and Sutliff is $-.62(p<.01)$. This means that goodness and similarity both predict average sorting times in that study. When the two patterns were similar to one another, the pairwise classification time was comparatively longer than when the patterns were dissimilar to one another. This similarity effect does not address the second, goodness, effect they reported.

\section{Study 1. Similarity Judgments and Goodness}

In Garner and Sutliff's (1974) study, good patterns were rated as different from each other and poor patterns were rated as similar. It is not necessary for good patterns (patterns from small R\&R sets) to be perceptually different from other patterns or for poor patterns (patterns from large $R \& R$ sets) to be similar. There are 581,400 possible 9-dot patterns in a 5 by 5 matrix when the subset is restricted to at least one dot in each row and column. This is the restriction commonly used to generate dot patterns in goodness studies. ${ }^{1}$ By computer-generating some of these patterns, many small R\&R subset patterns, good patterns, were found which appeared similar to each other and many large R\&R subset patterns, poor patterns, were found which appeared distinctive. Four stimuli having subset size R\&R4 (good patterns) and four having subset size R\&R8 (poor patterns) were selected for study. These patterns are shown in Figure 2.

Observers judged these eight stimuli for similarity, and the average judgments were scaled by usual techniques (Takane, Young, \& deLeeuw, 1977). The resulting two-dimensional similarity solution is shown in Figure 1. Patterns $1-4$ are good; Patterns 5-8 are poor. The two-dimensional scaling solution provided a reasonably good fit to the similarity judgments: Stress 1 was .13. Stress 1 was .08 for the three-dimensional solution. As shown in Figure 1, the four good patterns (R\&R4s) are relatively similar, while the four poor patterns (R\&R8s) are each removed from the R\&R4s and the other R\&R8s. Poor Patterns 5 and 6 are fairly close to each other in this two-dimensional solution; however, these two patterns are well separated in the three-dimensional solution, while there is relatively little added separation of the R\&R4s. These good patterns are similar to each other and these poor patterns are each relatively isolated in similarity.

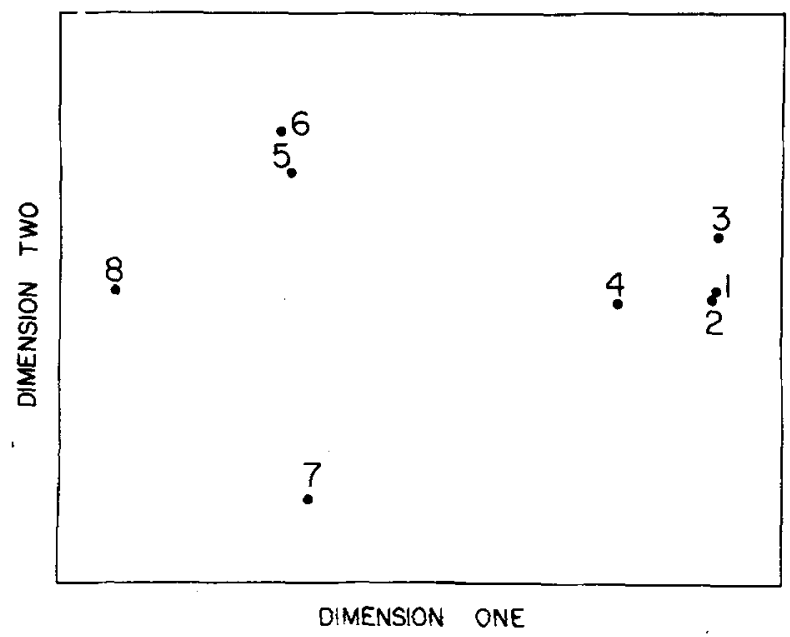

Figure 1. The two-dimensional similarity solution for the eight stimuli in Study 1. 
The similarity scaling solution shown in Figure 1 might suggest that the patterns selected for study are not good patterns by any criterion other than the physical rotation and reflection measure. Garner and Clement (1963) showed that factors other than $R \& R$ group size relate to goodness. Two such measures are reported in the next two studies, goodness judgments and free classification behavior.

\section{Study 2. Pattern Goodness}

Twelve observers rated each pattern in Figure 2 for goodness. The instructions were, after a brief examination of all eight patterns, to assign a number from 1 , for very good, to 7 , for very poor, to each randomly presented pattern. The means of these ratings are shown in Figure 2. The patterns belonging to small $R \& R$ groups are judged to be of greater goodness than are the patterns belonging to large $R \& R$ groups. This result is consistent with the findings of Garner and Clement (1963) and Glushko (1975). Patterns belonging to small R\&R subsets are rated as good.

\section{Study 3. Free Classification}

The same 12 observers used in Study 2 were asked to group the eight patterns shown in Figure 2 into at least two, but not more than seven, groups by any criterion they wished.

The observers used from 4 to 6 (mean $=4.5$ ) categories. The four good patterns were generally classified together. Patterns 5, 6, and 8 were sometimes classified together. Pattern 7 was always classed separately, except for one time when it was placed with Pattern 4. These clusterings are consistent with the similarity differences in Figure 1. There, too, the R\&R4 patterns are near each other; Patterns 5, 6, and 8 are also near each other but much less so; Pattern 7 is relatively isolated. This free classification outcome is consistent with the similarity scaling results. This result is also consistent with the classification report of Garner and Clement (1963) that patterns of the same $R \& R$ group size tend to be clustered together in a free classification task; however, this may not be a general rule.

\section{Study 4. Focusing Performance}

Several reports in the literature have concluded that good patterns can be processed more rapidly than poor patterns (Bell \& Handel, 1976; Checkosky \& Whitlock, 1973; Clement \& Varnadoe, 1967; Garner \& Sutliff, 1974; Pomerantz, 1977). The underlying basis for the goodness effect has been a matter of theoretical interest. Some evidence (Bell \& Handel, 1976; Garner \& Sutliff, 1974) suggests that good patterns can be encoded more rapidly than poor patterns. Other evidence suggests that memory effects are the source of the advantage (Checkosky \&
Whitlock, 1973; Pomerantz, 1977). In general, it has been offered that patterns of small R\&R subset size are good and that good patterns are easily processed because they can be rapidly encoded or better remembered.

It has also been reported that dissimilar patterns are readily classified, while similar patterns are confusable and difficult to uniquely categorize (Checkosky \& Whitlock, 1973; Lockhead, 1970; Lockhead \& King, 1977). We have seen that goodness and similarity are not always correlated in the same way. Because of this fact, goodness and similarity predictions can conflict. According to the hypothesis that good patterns can be easily focused, Patterns 1-4 in Figure 2 should be easily classified because they are good, while Patterns 5-8 should be more difficult because they are poor. According to the similarity hypothesis, Patterns $5-8$ should be easy to classify because they are more distinct and Patterns 1-4 should be difficult because they are near other patterns in the similarity space. These predictions are evaluated in the following experiment.

\section{METHOD}

Subject

Eight undergraduates served as observers as a partial requirement for an undergraduate psychology course.

\section{Stimuli}

The stimuli were the eight 9-dot patterns shown in Figure 2.

\section{Apparatus}

The patterns were presented on a $16 \times 22 \mathrm{~cm}$ Tektronix 611 oscilloscope, approximately $90 \mathrm{~cm}$ from the observer, in a dimly illuminated and sound-attenuating room. The patterns were written on the screen in less than $5 \mathrm{msec}$. Each pattern subtended approximately $.5^{\circ} \times .5^{\circ}$ of visual angle.

\section{Procedure}

There were eight blocks of 146 trials each. At the beginning of the experiment, the observer studied all eight patterns. For each block of trials, the observer was given a replica of one of the patterns and was instructed that this pattern would be the focused stimulus (the target) for that block. Each time the tar-

\begin{tabular}{|c|c|c|c|c|c|}
\hline NUMBER & 1 & 2 & 3 & 4 & \multirow{3}{*}{ RQR 4} \\
\hline PAT TERN & & & & $\because$ & \\
\hline GOODNESS & 2.75 & 2.08 & $2 \cdot 25$ & $2 \cdot 42$ & \\
\hline NUMBER & 5 & 6 & 7 & 8 & \multirow{3}{*}{ R\&R 8} \\
\hline PATTERN & : & & & & \\
\hline GOODNESS & 5.08 & 5.50 & 4.75 & 5.67 & \\
\hline
\end{tabular}

Figure 2. The eight stimulus patterns and their associated average goodness ratings obtained in Study 2. 
get was presented, the observer was instructed to press the key under the index finger of the preferred hand as quickly as possible, while attempting not to make errors. If one of the remaining seven patterns (a nontarget) was presented, the key under the index finger of the nonpreferred hand was to be pressed.

The subjects were informed that the target would occur on a random $50 \%$ of the trials and that the other seven stimuli would occur equally often on the other half of the trials. If an incorrect response was made, a large " $X$ " appeared above the pattern, and the pattern remained on the screen for $2 \mathrm{sec}$. If the correct response had been given, the screen erased immediately and the next stimulus appeared in $2 \mathrm{sec}$. For each of the eight blocks of trials, a different pattern was selected as the target. The order in which each pattern was the target was balanced across observers in a Latin square design. After every two blocks of trials, each observer had a 5-min rest period. Data from the first 20 trials of each block were considered practice and discarded.

\section{RESULTS}

The mean response times and the mean error rates to the target stimuli were positively correlated $(r=$ $.86, \mathrm{p}<.01$ ), indicating that there was no speedaccuracy tradeoff. The average error rate to target stimuli was $3.8 \%$; the average error rate to nontarget stimuli was $7.8 \%$. This difference in error rate, depending on whether the stimulus was a target or nontarget, suggests that the subjects tended toward reporting "yes." In all cases considered, errors and response times were positively correlated. Only response time data are presented here; any conclusions made with response times seem appropriate to errors as well, although there were too few errors in some comparisons to allow demonstration of statistical differences.

The average results relating $R \& R$ set membership or goodness to performance are reported first. To determine the effect of group membership (good or poor) on performance, responses were averaged over patterns belonging to the same goodness class. Figure 3 shows the mean response times, depending on whether the target and nontarget were good (R\&R4) or poor (R\&R8) patterns. Target (yes) and nontarget (no) response times were evaluated in separate variance analyses. "Yes" responses (open circles in Figure 3) were faster to poor targets than to good targets [413 vs. $571 \mathrm{msec} ; \mathrm{F}(1,7)=31.28, \mathrm{p}<.001]$. "No" responses (filled indices in Figure 3 ) depended on the group (good or poor) to which the target belonged $[F(1,7)=55.08, p<.001]$ and on the nontarget group (good or poor) to which the stimulus belonged $[F(1,7)=123.27, p<.001]$.

The pattern of results in Figure 3 is consistent with the similarity relations shown in Figure 1 and with the predictions of the similarity hypothesis. According to the similarity hypothesis, positive responses to targets should be fast when the targets are isolated from nontarget stimuli in similarity, and positive responses should be slow when the targets are similar to other nontargets. As shown by the open circles in Figure 3, the poor patterns that are isolated in similarity (Figure 1) are much easier to respond "yes" to than are the good targets which are not isolated in similarity.

Nontarget response times are also a function of the similarity relations shown in Figure 1 . When the target is a poor pattern, all nontargets are responded to rapidly and with about the same latency. This result is predicted by the fact that all the alternative patterns are removed in similarity from every poor pattern. When the target is a good pattern, responses to poor stimulus patterns that are dissimilar to the good target (Figure 1) are fast. However, responses to the remaining nontargets (the other good patterns), which are very similar to the good target pattern, are very slow. Again, this result follows from the similarity relations described in Figure 1.

These response latencies to target and nontarget stimuli as a function of stimulus goodness do not support the focusing hypothesis. By the focusing suggestion, good stimuli should be faster classified targets than poor stimuli. This is directly opposite to the result found here. Also, by the focusing suggestion, nontarget response times should never be faster than positive responses; but they are here. There seems to be no evidence in these data that the subjects used a go/no-go response strategy.

The similarity hypothesis cannot predict the additional finding that positive responses were often faster than negative responses. This result appears in

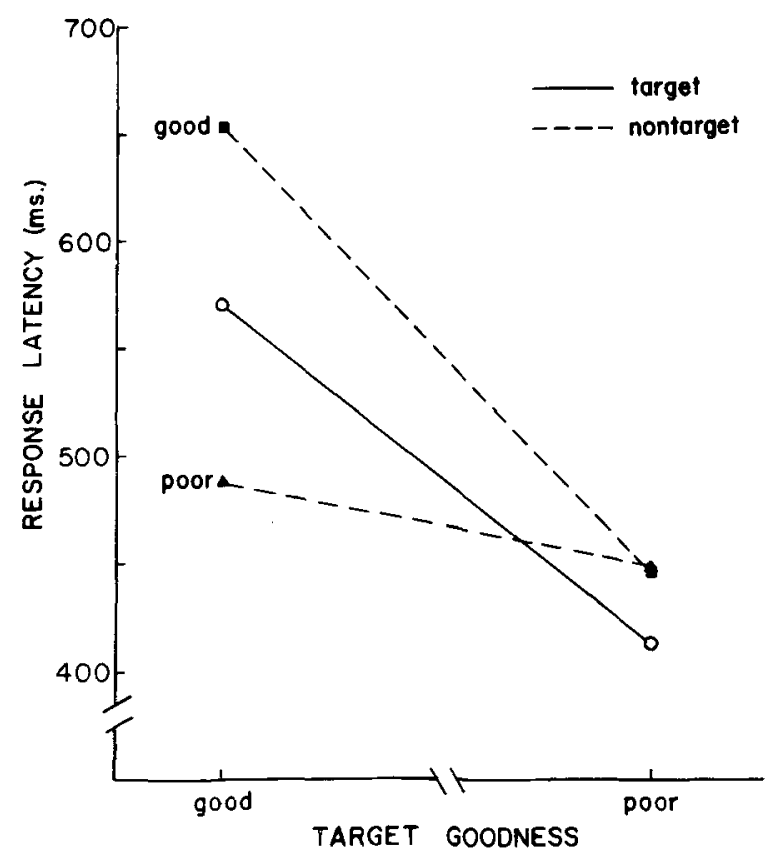

Figure 3. Mean response latencies as a function of target goodness from Study 4. 
Figure 3 when the target was poor, and is seen again when the target was good and the nontargets were also good. This may be a procedural result. Target stimuli in this experiment occurred on half the trials, and the nontargets occurred equally often on the other half of the trials. Thus, given that a target had occurred, the same pattern repeated on the successive trial an average of half the time. The comparable statistic for each nontarget stimulus was only 7\%. It is commonly the case that responses are facilitated on trials that the object repeats (Lockhead, Gruenewald, \& King, 1978); this may account for the fact that these "yes" responses are faster than the "no" responses. Furthermore, repetition effects become large when the discrimination task is difficult (Biederman, 1972); this may account for the additional fact that there is a larger advantage for the "yes" responses when the task is difficult (good targets, good stimuli) than when the task is easy (see Figure 3).

Response times to the individual stimuli are considered next. The mean response time to each stimulus target is shown in column 2 of Table 1. According to an analysis of variance (Target Stimulus by Subjects), mean response time depends on the target $[\mathrm{F}(7,49)=11.73, \mathrm{p}<.001]$. According to a Newman-Keuls contrast test, at alpha $=.05$, Target Stimulus 3 was responded to more slowly than any other target; Target 2 was identified more slowly than any of the poor patterns (5-8); and Targets 1 and 4 were classified more slowly than were Patterns 5 , 7 , or 8 . At alpha $=.06$, every good target was classified more slowly than was any poor target, and no difference between any of the poor targets was reliable.

Table 1 presents the relations among the response times, the similarity measures, and the goodness measures. In Columns 3-6 of Table 1 are four measures from the similarity judgments taken on the same stimuli as reported in Study 1. In column 7 are the goodness ratings from Study 2.

The similarity measures in columns 3 and 4 are from the three-dimensional solution of the similarity judgment data according to the scaling algorithm; the measures in columns 5 and 6 are the average raw similarity judgments provided by the subjects; these data avoid any artifactual problems that use of the scaling algorithm might generate. The similarity distance called B (columns 3 and 5) is the average distance in similarity between the target stimulus and all other stimuli in the set; this is a measure of the discriminability between events (Gruenewald, 1978). The similarity distance called LD (columns 4 and 6) is the least distance. This is the distance in similarity between the target and the nearest other stimulus in the set; this measure has been useful in
Table 1

Relations Among Response Times, Similarity Measures, and Goodness Measures

\begin{tabular}{rrrrrrr}
\multicolumn{7}{c}{ and Goodness Measures } \\
\cline { 3 - 7 } 1 & 2 & 3 & 4 & 5 & 6 & 7 \\
\hline 1 & 532 & .739 & .239 & 5.56 & 2.45 & 2.75 \\
2 & 561 & .692 & .139 & 5.55 & 1.82 & 2.08 \\
3 & 650 & .708 & .139 & 5.31 & 1.82 & 2.25 \\
4 & 542 & .652 & .179 & 5.47 & 2.55 & 2.42 \\
5 & 427 & .861 & .536 & 6.60 & 5.18 & 5.08 \\
6 & 453 & 1.000 & .600 & 7.22 & 5.18 & 5.50 \\
7 & 402 & 1.170 & 1.063 & 8.13 & 7.55 & 4.75 \\
8 & 382 & .995 & .536 & 7.31 & 5.55 & 5.67 \\
\hline
\end{tabular}

Note-Column $1=$ stimulus number from Figure 2, Column $2=$ mean response latency to focus each stimulus from Study 4, Columns 3, 4, 5, and $6=$ four measures of relationship between stimuli (Scale B, Scale $L D$, judged $B$, and judged $L D$, respectively; see text for description), and Column $7=$ mean goodness ratings from Study 2. Correlations between response latency and each measure are: $r_{23}=-.82, r_{24}=-.81, r_{25}=-.88, r_{26}=-.90$, $r_{27}=-.90$.

predicting word classification times (Hutchinson \& Lockhead, 1977).

The dependent measures in Table 1 are all interrelated, and each correlates significantly $(p<.01)$ with response time to classify the target. These correlations range from -.81 to -.90 and are shown at the bottom of the table. There is no way from the present data to select any one predictor over any other for determining response times. The time to classify the target item increases with increasing similarity between that target and the nearest nontarget (LD). Response time also increases with increasing similarity between that target and the average distance of all nontargets (B). These conclusions hold whether the similarity numbers chosen come from the raw judgments or from the algorithm.

The time to classify the target varies inversely with rated goodness of the target pattern $(r=-.90)$. This is the reverse of the result normally reported in the literature. Patterns which are symmetric (small $\mathbf{R} \& \mathbf{R}$ group membership) are rated as good, but whether or not these good patterns are easy to identify depends less, if at all, on their goodness than on their similarity to other patterns in the current stimulus set.

\section{CONCLUSIONS}

The primary results of this investigation are that similarity measures predict how readily particular stimuli can be responded to in a focusing task, and that poor patterns can be easier to classify than good patterns.

Patterns are rapidly and accurately classified if they are dissimilar from other patterns in the stimulus set. This is the case whether those patterns are rated 
as good and belong to small $R \& R$ subsets or rated as poor and belong to large $R \& R$ subsets. Performance improves as the similarity decreases between the target stimulus and stimuli which belong to the "no" response category. This is one measure showing that context influences performance. ${ }^{2}$ In a search task, as well as in other tasks (King et al., 1978), the similarity of possible alternatives to the sought target affects the ease with which people can respond positively to that target stimulus and negatively to other stimulus patterns.

Concerning how the focusing task is performed, it appears that people do not, at least not always, attend to some critical feature or aspect of the target stimulus and then respond "yes" when that feature is detected and "no" when it is decided that the sought attribute is not present. If they did, nontarget response times would not depend on the target. People were slow to respond "no" to a good pattern and fast to respond "no" to a poor pattern when the target was a good pattern. If the negative response was a default judgment about the presence of some feature, then this response time difference would not occur. According to feature detection hypotheses, the time to report the absence of a critical feature should not depend on noncritical features. For the same reason, it is also concluded that the observers do not search for the entire target stimulus or configuration and respond on the basis of whether that configuration was presented. There is logically no difference between searching for a feature, for a configuration, or for the whole stimulus to be matched. Each of these cases is the search for some kind of a template to match. Any process based only on characteristics of the focused stimulus does not account for these data.

Focusing performance in the present experiment has analogies to an identification process. It appears as if the subject identifies a presented stimulus pattern and interrogates memory as to whether or not this is the sought pattern. It has been shown that the identification process is subject to strong contextual influences from alternative stimuli (Monahan \& Lockhead, 1977). That finding is reinforced by the present result. Patterns similar to other patterns in the set are difficult to discriminate and hence to identify, whether they are targets or nontargets.

The influence of the pattern goodness of the stimulus on the identification process is less clear. Certainly any effects of goodness can be overwhelmed by the similarity relations among alternative stimuli. This is not, however, to say that stimulus factors, such as pattern goodness, have nothing to do with performance. It is to say that, whatever role pattern goodness may have in information processing, stimulus set aspects must also be considered in describing performance in focusing tasks, in particular, or classification tasks, in general.
The conclusions just noted help to discriminate between two classes of theory, that class in which the stimulus, or some feature of the stimulus, is treated as a template or configuration to be searched and that class in which the stimulus is hypothesized to be initially perceived in some general location in perceptual space. In this second class of model (Lockhead, 1972), what is essential is whatever it is that similarity measures. Specific features or templates are not essential. Context, defined by this theory as the psychological differences or discriminabilities among items in the stimulus set, primarily determines performance.

\section{REFERENCES}

Bell, H. H., \& Handel, S. The role of pattern goodness in the reproduction of backward masked patterns. Journal of Experimental Psychology: Human Perception and Performance, $1976,2,139-150$

Biederman, I. Human performance in contingent informationprocessing tasks. Journal of Experimental Psychology, 1972, 93, 219-238.

Checkosky, S. F., \& Whitlock, D. Effects of pattern goodness on recognition time in a memory search task. Journal of Experimental Psychology, 1973, 100, 341-348.

Clement, D. E., \& Varnadoe, K. W. Pattern uncertainty and the discrimination of visual patterns. Perception \& Psychophysics, 1967, 2, 427-431.

GARNER, W. R. The processing of information and structure. Potomac, Md: Erlbaum, 1974.

Garner, W. R. Selective attention to attributes and to stimuli. Journal of Experimental Psychology: General, 1978, 107, 287. 308.

Garner, W. R., \& Czement, D. E. Goodness of pattern and pattern uncertainty. Journal of Verbal Learning and Verbal Behavior, 1963, 2, 446-452.

Garner, W. R., \& Suthiff, D. The effect of goodness on encoding time in visual pattern discrimination. Perception \& Psychophysics, 1974, 16, 426-430.

Glushko, R. J. Pattern goodness and redundancy revisited: Multidimensional scaling and hierarchical clustering analyses. Perception \& Psychophysics, 1975, 17, 158-162.

GrUENEWALD, P. G. The classification of multidimensional stimuli. Unpublished doctoral dissertation, Duke University, 1978.

Hutchinson, J. W., \& Lockhead, G. R. Similarity as distance: A structural principle for semantic memory. Journal of Experimental Psychology: Human Learning and Memory, 1977, 3, 660-678.

King, M. C., Gruenewald, P. J., \& Lockhead, G. R. Classifying related stimuli. Journal of Experimental Psychology: Human Learning and Memory. 1978, 4, 417-427.

LockHeAD, G. $R$. Identification and the form of multidimensional discrimination space. Journal of Experimental Psychology, 1970, 85, 1-10.

Lockhead, G. R. Processing dimensional stimuli; A note. Psychological Review, 1972, 79, 410-419.

Lockнead, G. R., \& KING, M. C. Classifying integral stimuli. Journal of Experimental Psychology: Human Perception and Performance, 1977, 3, 436-443.

Lockhead, G. R., Gruenewald, P. G., \& King, M. C. Holistic vs. attribute repetition effects in classifying stimuli. Memory \& Cognition, 1978, 6, 438-445.

Monahan, J. S., \& Lockhead, G. R. Identification of integral stimuli. Journal of Experimental Psychology: General, 1977, 106, 94-110. 
Pomerantz, J. R. Pattern goodness and speed of encoding. Memory \& Cognition, 1977, 5, 235-241.

Takane, Y., Young, F. W., \& de Leeuw, J. Nonmetric individual differences multidimensional scaling: An alternating least squares method with optimal scaling features. Psychometrika, 1977, $42,7-67$.

\section{NOTES}

1. Rotation and reflection criteria of pattern goodness were developed (see Garner, 1974) as a physical measure of the symmetry in dot patterns. Consider the 90 patterns which can be constructed by placing dots in five cells of a 3 by 3 matrix with the constraint of a dot in each row and column. Any pattern which is not symmetric about either the horizontal or the vertical axis in this matrix can be rotated (in $90^{\circ}$ steps) or reflected (in $180^{\circ}$ steps), and each transformation produces a new pattern. There are eight such alternatives to each of these patterns, and each such pattern is called an $R \& R 8$. A pattern that is symmetric about either the horizontal or the vertical axis, but not both, produces only four unique patterns by these translations. Each of these is a member of a rotation and reflection group of four alternatives, called an R\&R4. An example of an R\&R4 pattern is when there is a dot in each cell of the bottom row and the leftmost column of the matrix; this pattern looks like the letter "L." Patterns which are symmetric about both the horizontal and vertical axes exactly repeat themselves on all eight transformations. There are only two such 5-dot patterns, which belong to R\&R1 subsets; one of these looks like a + and the other like an $\times$. Patterns which belong to small subsets are rated as good. The larger the subset to which a pattern belongs, the poorer that pattern is rated to be (Garner \& Clement, 1963).

2. In Pomerantz's (1977) study, however, context was not important to performance. This discrepancy with our results might be related to three procedural differences between Pomerantz's experiment and ours: his stimuli subtended $3^{\circ}$ of visual angle and were thus only marginally within the fovea, while our stimuli subtended $.5^{\circ}$; his nontarget sets contained only 2 or 4 items, while ours contained 7; and he gave his observers samples of all the task patterns to keep beside the response keys, while we did not. Pomerantz's observers might sometimes have performed in the following manner. First, perhaps they studied the patterns to discover some distinguishing characteristic(s). This should be easy; there were few stimuli in a task, often only two dot locations were common to a pair of stimuli, and the patterns were available simultaneously for this inspection. Then the subjects may have attended to that selected discriminating section of the display and responded with a go/no-go strategy, depending on whether the discriminating light(s) was (were) illuminated when the pattern came on; with about $36^{\prime}$ of visual angle between the nearest two lights, this could have been easy. Indeed, it may be relatively difficult for people to immediately apprehend nine dots spread over $3^{\circ}$ as a pattern. That is, a go/no-go strategy may have been used in Pomerantz's study, and the observers may also have not used all of the stimulus pattern.

A related concern is why a good pattern is responded to relatively faster than a poor pattern when there are only two patterns in the task (Garner \& Sutliff, 1974). If the above guess about performance in Pomerantz's study is correct, then this goodness advantage may also be explicable. Garner and Sutliff also used $3^{\circ}$ patterns. If observers find a critical light to attend to, the perceptible structure in a good pattern may make it easier for them to remember where that light is. By this conjecture, and conjecture it is, subjects do different things when there are large patterns with few alternatives (Garner \& Sutliff, 1974; Pomerantz, 1977) than they do with more compact patterns and several alternatives (our Study 4). They may sometimes process single lights when patterns are very large (they would have to if the patterns were made much larger), and they may more often process the entire pattern when all elements are close together and many patterns are in the set.

(Received for publication April 24, 1979; revision accepted August 17, 1979.) 